\title{
Added Value of Semi-quantitative Analysis in Interim FDG-PET/CT in Pediatric Lymphoma
}

\author{
Hussein, Sh and Moustafa, $H$ \\ Nuclear Medicine Unit (NEMROCK), Faculty of Medicine, Cairo University, Egypt.
}

Pediatric lymphoma (PL) is one of the few pediatric malignancies that shares aspects of its biology and natural history with adult lymphoma. However, it differs from the adult counterparts, mainly in terms of histopathology and therapeutic strategies (1). It comprises 6\% of all childhood cancers worldwide (2). In Egypt, childhood lymphoma represents $1.3 \%$ of all incident cancers and $28.7 \%$ of all childhood cancer occupying the second rank among all childhood malignancies (3).

PL is highly sensitive to standard chemotherapy, radiation therapy, or combined-modality therapy (4). Approximately 90-95\% of PL can be cured with 5-year survival rate of around 96\%; prompting increased attention to the long-term morbidity for these patients (5).

The conventional anatomic imaging for early treatment response monitoring is based on the change in tumor size, which is not an accurate predictor of outcome (6), however, functional assessment of response using FDG-PET performed early after two cycles of therapy (interim PET) represents a useful non-invasive imaging method to monitor the treatment benefits in adulthood and pediatric lymphomas (7). Moreover, it has been demonstrated to predict therapy outcome at an earlier stage of treatment allowing a risk-adapted treatment strategy (8).

The Imaging Subcommittee of the "International Harmonization Project in Lymphoma" has defined criteria for PET interpretation after completion of chemotherapy (9); that cannot be applied in interim PET that needs special criteria to assess response (10).These criteria have not been sufficiently validated, especially in pediatric patients.

Practically speaking; the methods of data analysis used have been broadly categorized into two groups: (1) qualitative analysis, i.e. visual assessment; (2) semi-quantitative analysis, i.e., standardized uptake value (SUV) and recently, volume-based metabolic parameters.

Corresponding Author: Hussein, Sh. Email: shimos81@yahoo.com. 


\section{Editorial}

\section{1-Qualitative (visual) assessment:}

In 2009, the 1st international workshop on interim PET in lymphoma was held in Menton, France and it resulted in so-called "Deauville criteria" recommendation (10). Deauville criteria propose simple, reproducible rules for visual interpretation of interim PET in malignant lymphomas, these criteria were contained in three major statements: (i) Visual assessment is preferred, but SUV determination can be used in some cases; (ii) Interim-PET interpretation should always be made by comparing the foci of FDG uptake to those recorded in the baseline study; (iii) The intensity of FDG uptake should be graded according to a five-point scale in which a reference organs should be the mediastinum and the liver, are used to define different grades of FDG uptake (10).

"Minimal residual uptake" (MRU) is a term which has been used to describe low-grade uptake that may be seen after treatment for lymphoma (11). The significance of MRU in interim PET scans may differ according to the lymphoma type (e.g. HL vs. NHL), stage (early vs. advanced) and possibly the treatment (12). In patients with early HL receiving ABVD chemotherapy and involved field radiotherapy with a low pretest likelihood of disease, MRU is associated with a very good prognosis. Conversely MRU appears to be associated with a poor prognosis in patients with advanced NHL receiving systemic chemotherapy and a higher pretest likelihood of disease $(\mathbf{1 1}, \mathbf{1 2})$.

\section{2- Semi-quantitative assessment:}

\section{A-The Standardized uptake value (SUV):}

It is calculated from the counts-per-pixel and normalized to body weight (BW) (13), using the following formulas:

SUV $_{\text {BW }}=$ Tissue activity $(\mathrm{KBq} / \mathrm{ml}) /$ Injected activity* (MBq)/weight (Kg)

Where *activity was decay-corrected from the delay between injection and image acquisition.

SUVmax is the count in the most active pixel in the VOI.

SUVmean is the average of the counts in all pixels in the Volume of Interest (VOI).

$\Delta$ SUVmax is the percent of change between SUVmax on the PET2 scan (interm PET) versus the PET1 scan (initial PET). This method of assessing metabolic response has been recommended by the 1999 European Organization for Research and Treatment of Cancer (EORTC) position paper on measurement of metabolic tumor response with FDG (14). It is calculated using the following formula; $\Delta$ SUV max $=($ SUV $\max 1-S U V \max 2) /$

\section{SUV max1) X 100}

SUVmax is proved to improve the prognostic value of early ${ }^{18} \mathrm{~F}$-FDG $\mathrm{PET} / \mathrm{CT}$ when it is added to a visual analysis (15); however, in some studies SUVmean may be potentially more valuable. This may be attributed to that a single voxel value may not be representative of the overall tumor uptake in a non homogeneous tumor (16).

SUV normalized to body surface area $\left(\mathrm{SUV}_{\mathrm{bsa}}\right)$ or lean body mass (SUL) could be more precise 


\section{Editorial}

in pediatric population than SUV normalized to body weight $\left(\mathrm{SUV}_{\mathrm{BW}}\right)$, as they are almost independent of body parameters in the pediatric population and would serve as better metabolic activity markers (17).

\section{B- Volume-based metabolic parameters:}

Such as metabolic tumor volume (MTV) and total lesion glycolysis (TLG) have become potentially important semi-quantitative PET indices (18).

MTV was measured by generating 3D iso-count contours to define tumor boundaries (VOI) using semi-automatic contouring software after applying a pre-defined threshold of the SUVmax value within this VOI, this may fail in case of low tumor-to-background ratios or in the vicinity of organs showing high uptake (bladder, heart, brain, kidneys), in these cases the automatically generated VOI should be visually checked and manually drawn so it will be large enough to include all the tumor volume and careful enough to exclude areas of physiological uptake (19). $\Delta$ MTV is calculated as the percent of change between MTV on the PET2 scan versus the PET1 scan using the following formula;

\section{(MTV1 - MTV2)/MTV1) X 100}

TLG was calculated by multiplying the selected PET volume (MTV) on the investigated lesions as mentioned above by the SUVmean within that volume: $\mathbf{T L G}=\mathbf{M T V} \mathbf{X}$ SUVmean

$\Delta$ TLG: is calculated according to LarsonGinsberg Index (LGI), and known as $\triangle \mathrm{TLG}$ (LGI) (18):
$\left.\left[(\mathrm{SUVmean})_{1} \mathrm{X}(\mathrm{Vol})_{1} \text { - (SUVmean) }\right)_{2} \mathrm{X}(\mathrm{Vol})_{2}\right] /$ (SUVmean) ${ }_{1} X\left(\right.$ Vol) ${ }_{1} X 100$

These volume based parameters are theoretically more relevant parameters than are single pixel values (17) They were thought to provide valuable information regarding tumor aggressiveness and consequently, they may be considered as potential prognostic indices for tumors $(\mathbf{2 0}, \mathbf{2 1}, 22,23)$.

The main obstacle to the wide implementation of TLG as a parameter for assessing response is the method used to measure the MTV which is considered as a combined metabolic radiological marker. The choice of the thresholding method for MTV may affect the absolute value of the MTV (24). Multiple studies demonstrate that the best thresholding methods are either an SUV within the range (2-3) or a fixed threshold of 40 $\%$ of the SUVmax as this approximates tumor volume best and are optimal for differentiating benign from malignant lesions, and minimizes inclusion of unwanted physiological FDG uptake in normal tissues (25). However, other studies have demonstrated that one specific adaptive threshold for determination of MTV, either fixed SUV cut-off or percentage threshold, will not yield volumes exactly corresponding to those produced by manual contouring of CT scans nor to those obtained from pathology, while individualizing MTV calculation to tumor size and SUVmax may be worthwhile to optimize its prognostic stratifying value (26). 


\section{Editorial}

\section{REFERENCES:}

1- Pastore G, Magnani C, Verdecchia A, et al.: Survival of childhood lymphomas in Europe, 1978-1992: a report from the EUROCARE study. Eur J Cancer 2001; 37:703710.

\section{2- $\quad$ Ries L, Harkins D, Krapcho M, et al.:} SEER Cancer Statistics Review, 1975-2003. Bethesda, Md: National Cancer Institute, 2006.

3- Ibrahim AS, Seifeldin IA, Ismail K, et al.: Cancer in Egypt, Gharbiah: Triennial Report of 2000-2002, Gharbiah Population-based Cancer Registry. Cairo: Middle East Cancer Consortium; 2007.

4- Brepoels L, Stroobants S and Verhoef G: PET and PET/CT for response evaluation in lymphoma: current practice and developments. Leuk Lymphoma. 2007; 48: 270-282.

5- Robert J. Arceci, Louis S. Constine, Thomas G. Gross, et al.: National Cancer Institute: PDQ ${ }^{\circledR} \quad$ Childhood Non-Hodgkin Lymphoma Treatment (2012);http://cancer.gov/cancertopics/pdq/treatm ent/childnonhodgkins/HealthProfessional.

6- Rankin SC: Assessment of response to therapy using conventional imaging. Eur J Nucl Med Mol Imaging. 2003; 30(suppl 1):S56-S64.
7- Dann EJ, Bar-Shalom R, Tamir A, et al.: Risk-adap ted BEACOPP regimen can reduce the cumulative dose of chemotherapy for standard and high-risk Hodgkin lymphoma with no impairment of outcome. Blood. 2007; 109:905-909.

8- Kasamon Yvette L, Jones Richard J and Wahl Richard L: Integrating PET and $\mathrm{PET} / \mathrm{CT}$ into the risk-adapted therapy of lymphoma. J Nucl Med. 2007; (48) (Suppl. 1):19S-27S.

9- Juweid ME, Stroobants S, Hoekstra OS et al.: Use of positron emission tomography for response assessment of lymphoma: consensus of the Imaging Subcommittee of International Harmonization Project in Lymphoma. J Clin Oncol 2007; 25: 571-578.

10- Meignan M, Gallamini A, and Haioun C.: Report on the first international workshop on interim-PET scan in lymphoma. Leuk Lymphoma 2009; 17: 1-4.

11- Mikhaeel NG, Hutchings M, Fields PA, et al.: FDG-PET after two to three cycles of chemotherapy predicts progression-free and overall survival in high-grade non-Hodgkin lymphoma. Ann Oncol. 2005; 16: 1514-1523. 


\section{Editorial}

12- Hutchings M, Loft A, Hansen M, et al.: FDG-PET after two cycles of chemotherapy predicts treatment failure and progression-free survival in Hodgkin lymphoma. Blood. 2006; 107: 52-59.

13- Graham MM, Peterson LM and Hayward RM: Comparison of simplified quantitative analyses of FDG uptake. Nucl Med Biol. 2000; 27: 647-655.

14- Young H, Baum R, Cremerius U, Herholz K, Hoekstra O, Lammertsma AA, Pruim $\mathbf{J}$ and Price P: Measurement of clinical and subclinical tumour response Using [18-F]fluorodeoxyglucose and positron emission tomography: Review and 1999 EORTC Recommendations. Eur J Cancer, 1999. 35(13): p. $1773-82$.

15- Lin Chieh, Itti Emmanuel, Haioun Corinne, et al.: Early ${ }^{18} \mathrm{~F}-\mathrm{FDG}$ PET for Prediction of Prognosis in Patients with Diffuse Large B-Cell Lymphoma: SUV-Based Assessment versus Visual Analysis. J Nucl Med 2007; 48:1626-1632 DOI:

10.2967/jnumed.107.042093.

16- Soret M, Bacharach SL and Buvat I.: Partial-volume effect in PET tumor imaging. $\mathrm{J}$ Nucl Med 2007; 48: 932-945.

17- Yeun HW, Sanches A, Squire OD, Macapinlac HA, Larson SM, Erdi YE
Standardized uptake value in pediatric patients: an investigation to determine the optimum measurement parameter. Eur J Nucl Med (2002) 29: 61-66.

18- Larson SM, Erdi Y, Akhurst T, et al: Tumor treatment response based on visual and quantitative changes in global tumor glycolysis using PET-FDG imaging. The visual response score and the change in total lesion glycolysis. Clin Positron Imaging 2:159-171, 1999.

19- Boellaard R, O'Doherty MJ, Weber WA, Mottaghy FM, Lonsdale MN, Stroobants SG, et al.:FDG PET and PET/CT: EANM procedure guidelines for tumour PET imaging: version 1.0. Eur J Nucl Med Mol Imaging. 2010; 37(1):181-200.

20- Seung Hwan Moon, Joon Young Choi, Hwan Joo Lee, et al.: Prognostic Value Of ${ }^{18} \mathrm{~F}$ FDG- PET/CT In Patients With Squamous Cell Carcinoma Of The Tonsil: Comparisons of Volume-Based Metabolic Parameters Published online in Wiley Online Library (wileyonlinelibrary.com 2011, DOI: 10.1002/hed.22904.

21- Abd El-Hafez YG, Moustafa HM, Khalil HF, Liao CT and Yen TC: Total lesion glycolysis: A possible new prognostic parameter in oral cavity squamous cell carcinoma. Oral Oncol(2012),http://dx.doi.org/10.1016/j.oralonc ology.2012.09.005. 


\section{Editorial}

22- Sharma P, Gupta A, Patel C, et al.:

Pediatric lymphoma: metabolic tumor burden as a quantitative index for treatment response evaluation. Ann Nucl Med. 2012; 26(1):58-66.

23- Tseng D, Rachakonda LP, Su Z, et

al.: Interim-treatment quantitative PET parameters predict progression and death among patients with Hodgkin's disease. Radiat Oncol. 2012; 19: 7- 5.

24- Cheebsumon $\mathbf{P}$, van Velden FH, Yaqub M, H et al.: Measurement of metabolic tumor volume: static versus dynamic FDG scans. EJNMMI Res. 2011; 1.35.
25- Van de Wiele Christophe, Kruse Vibeke, Smeets Peter, et al.: Predictive and prognostic value of metabolic tumour volume and total lesion glycolysis in solid tumours. Eur J Nucl Med Mol Imaging. 2013 Jan; 40(2):290-301.

26- Hyun SH, Choi JY, Shim YM, et al.: Prognostic value of metabolic tumor volume measured by ${ }^{18} \mathrm{~F}$-fluorodeoxyglucose positron emission tomography in patients with esophageal carcinoma. Ann Surg Oncol. 2010; 17:115-22. 